\title{
Costos de la Renovación Curricular. Una Propuesta Metodológica para la Valorización Económica de Carreras Universitarias
}

\author{
Luis Améstica-Rivas ${ }^{(1)}$, Xavier Llinas-Audet ${ }^{(2)}$ y Josep-Oriol Escardíbul( ${ }^{(3)}$ \\ (1) Departamento Gestión Empresarial, Facultad de Ciencias Empresariales, Universidad del Bío-Bío, \\ Casilla 447, Chillán, Chile. (e-mail: lamestica@ubiobio.cl) \\ (2) Cátedra UNESCO de Dirección Universitaria, Univ. Politécnica de Cataluña, Campus Nord - Edif. C5, \\ C. Jordi Girona, 1- 3, 08034 Barcelona, España. (e-mail: xavier.llinas@upc.edu) \\ (3) Depto. de Economía Pública, Economía Política y Economía Española, Fac. de Economía y Empresa, \\ Univ. de Barcelona e Instituto de Economía de Barcelona (IEB), España. (e-mail: oescardibul@ub.edu)
}

Recibido May. 30, 2016; Aceptado Ago. 1, 2016; Versión final Sep. 10, 2016, Publicado Feb. 2017

\begin{abstract}
Resumen
A partir del estudio de caso de una universidad pública chilena, se analiza la importancia de la renovación curricular en la gestión universitaria y se propone una metodología que sistematiza los costos y valoriza económicamente este proceso. Si bien los aspectos económico-financieros son estratégicos en la gestión universitaria, existe escasa literatura que los relacione a dichos procesos. Utilizando un análisis de corte y herramientas financieras, valor actual de costos (VAC), costo anual equivalente (CAE), simulación Monte Carlo y punto de equilibrio, se constata que en un contexto de autofinanciamiento, el punto de equilibrio de cada carrera se incrementa. Ante la inexistencia de apoyo extraordinario del Estado, para financiar los procesos de renovación curricular, las carreras deben aumentar en promedio seis estudiantes por cohorte 0 buscar nuevas fuentes de financiamiento. La renovación curricular exige disponer de los recursos para su implementación en cantidad y plazos definidos, para asegurar la sustentabilidad académica y financiera de la institución.
\end{abstract}

Palabras clave: renovación curricular; financiamiento universitario, gestión universitaria, innovación curricular, valorización económica.

\section{Curricular Renovation Costs. A Methodology for the Economic Valuation of University Degrees}

\section{Abstract}

The processes of curriculum renewal in universities have been built from the singularities of each institution. While economic and financial aspects are strategic in university management, there is a lack of research that relates these aspects with curriculum renewal processes.

From a study case of a Chilean public university, the importance of curriculum renewal in university management is analyzed and a methodology that systematizes costs and value economically this process is proposed. Using an analysis of cutting costs and financial tools, actual value cost (VAC), equivalent annual cost (CAE), Monte Carlo simulation, and balance point, it is found that in a context of self-financing, the balance point of each undergraduate program increases. In the absence of extraordinary support from the Government to finance the processes of curriculum renewal, undergraduate programs should increase an average of six students per cohort or look for new sources of funding. The curriculum renewal requires adequate resources for implementation in quantity and deadlines to ensure the academic and financial sustainability of the institution.

Keywords: curriculum renewal; university funding; university management; curriculum innovation; economic valuation 


\section{INTRODUCCIÓN}

Las universidades han asumido proyectos de renovación curricular con énfasis en metodologías docentes que posicionan al estudiante en el centro del proceso de aprendizaje. Asimismo, desarrollan proyectos de rediseño curricular basado en competencias y recogen la experiencia, aportes de egresados y de empleadores para alinear la formación profesional con las necesidades cambiantes y emergentes del mercado laboral (Clemente-Ricolfe y Escribá-Pérez, 2013; Hoogveld et al., 2003; Ion y Cano, 2012). Es así, como la implantación del Espacio Europeo de Educación Superior (EEES) ha promovido la metodología de la enseñanza enfocada en las competencias (Quevedo-Blasco et al., 2015), teniendo un importante protagonismo en la formación de profesionales (González et al., 2014; Tejada, 2012). Dicho proceso apunta hacia la competitividad y empleabilidad de los profesionales a partir de carreras fácilmente comparables y comprensibles (González y González, 2008). Para ello, se han reestructurado perfiles de egreso e incorporado sistemas de créditos transferibles en las universidades, comprometiéndose a vincular sus programas con el sector productivo y organizar los aprendizajes, de modo que resulten relevantes para la incorporación de sus egresados al mercado laboral (González y Wagenaar, 2003). En este sentido, López (2016), establece que el valor de la competencia reside en sus posibilidades de avance, integración y búsqueda continua de un saber integral e integrado que permite aprender y seguir aprendiendo en un escenario mundial y globalizado

Asimismo, se observa una creciente investigación concerniente a temas de currículo, especialmente en el ámbito de la teoría curricular y del diseño, así como una evolución en la conceptualización utilizada; por ejemplo, se tiende a sustituir los términos construcción curricular por gestión curricular (Márquez et al., 2010). Además, se enfatizan conceptos como innovación, cambio o transformaciones del currículo. Desde la perspectiva metodológica, se ha introducido el concepto de resultados de aprendizaje, como el conjunto de competencias que incluye conocimientos, comprensión y habilidades que se espera que el estudiante domine, comprenda y demuestre después de completar un proceso de aprendizaje (González y Wagenaar, 2003), en una perspectiva que va más allá de la aprobación de un currículo basado en objetivos cognitivos y que busca la aplicación de conocimientos en ambientes reales, abriendo la posibilidad de transformar experiencias de aprendizajes en la posesión de competencias (Schmal y Ruiz-Tagle, 2008).

Los cambios señalados suponen una transformación de la estructura institucional que implica desde el ingreso del estudiante a la universidad, su permanencia y por consiguiente genera todo un efecto en el sistema de valor que conlleva el egreso del estudiante. En este sentido, es importante conocer la cadena de valor de la universidad. Este último concepto que normalmente es aplicado a las empresas, también es extensible a organizaciones sin fines de lucro. Por lo tanto, las universidades como cualquier tipo de organización, necesita conocer el valor que provee en relación a aquellos que intervienen en sus procesos y financiación (McChlery et al., 2007).

\section{Renovación curricular y gestión universitaria}

El éxito de la renovación curricular en una universidad depende de las dinámicas organizacionales propias de cada institución y del nivel de involucramiento de los líderes. Algunos estudios establecen que en algunas instituciones no han habido cambios importantes porque se mantienen las formas tradicionales de enseñar y evaluar, así como normas alejadas del objetivo real de cambio (Díaz, 2006). Cabe destacar que el cambio exige capacidades de implementación y seguimiento de la reforma curricular, pero también del ámbito de la gestión. Es así como los procesos curriculares se llevan en un marco de tensión, dado los tiempos administrativos que demandan y así dimensionar los costos de las reformas y la forma de comunicar a los estudiantes (Icarte y Lávate, 2016).

En este sentido, se ha evidenciado que universidades han incrementado sus costos por estas renovaciones, siendo imprescindible que el Estado genere apoyos financieros extraordinarios para llevar acabo estos procesos, como reformar el currículo y disminuir la duración de los programas o incentivar cambios corporativos (Reich et al., 2011). Asimismo, existen costos ocultos que generan presiones en el interior de las universidades y que muchas veces lleva a las instituciones a subir las tasas y precios de sus carreras. La financiación es un factor clave para poner en marcha innovaciones en las universidades y condición sine qua non para que esta pueda tener éxito (Clark, 1998).

La problemática descrita, respecto a la disociación que se puede dar entre los gestores curriculares y los responsables de la gestión en las universidades, en términos de determinar la valorización económica de un proyecto tan estratégico para las instituciones, ha sido el detonante de esta publicación que viene a sistematizar y proponer una metodología que identifica los factores más relevantes que afectan a una universidad ante un proyecto de renovación curricular, evaluándola desde una perspectiva financiera. Para ello, se aplican dos métodos: por un lado, uno basado en la técnica de evaluación de proyecto del costo 
anual equivalente, denominado CAE (Riggs et al., 2002) con su correspondiente análisis de regresión; por otro lado, el punto de equilibrio desde una perspectiva de la estructura de costos (Horngren et al., 2007). El trabajo propuesto es coherente con el modelo de retorno de la inversión ROI de Phillips, para proyectos de formación y capacitación a partir del modelo Kirkpatrick y que es utilizado en algunas instituciones como referencial para evaluar programas de formación (Kirkpatrick y Hawk, 2006).

El aseguramiento de la calidad en la educación superior se ha convertido en un eje de constante preocupación para la agenda nacional en Chile. Para fortalecer la calidad algunas instituciones han implementado procesos de rediseño curricular como un criterio de selectividad y para la asignación de recursos internos y externos (Guzmán et al., 2015).

\section{Financiación y costos de la reforma curricular de las universidades}

Lograr una financiación eficiente es uno de los objetivos centrales que se pretende alcanzar en el sistema universitario, haciéndose imperativa la financiación de los modelos educativos que aseguren el éxito y, a su vez minimicen las necesidades económicas, en un contexto de racionalización de la gestión económica de las universidades y enfrentado en términos crecientes a altos niveles de competencia y reducción de la financiación por parte de los gobiernos (García, 2008; Martensen y Grønholdt, 2009; Solé y Llinàs-Audet, 2011). En este entorno, el "gerencialismo" en las universidades se constituye como uno de los principales estilos de gestión en la mayoría de países de la OCDE, es decir, asumir prácticas propias de la gestión empresarial en un modelo neoliberal con marcada retirada del Estado (Girotto et al., 2013).

Los procesos de renovación curricular se han instalado de distintas formas en las universidades en coherencia con sus proyectos educativos enunciados en sus planes de desarrollo estratégico. Estos procesos pueden ser más centralizados, desde la autoridad rectoral o con una conducción más compartida a nivel de las propias carreras o programas. En este contexto, es necesario operar el proyecto curricular dentro de una institución tomando en cuenta los recursos económicos, humanos, de infraestructura y equipamiento con el que cuenta la misma institución, área o unidad académica. Asimismo, también se deben de tener en cuenta los recursos necesarios que se tendrán que adquirir para poder operar las reformas sustentadas en el discurso de la innovación de los modelos educativos y proyecto curricular que se deseen alcanzar. Es importante precisar que el currículo es más que un producto o resultado final (plan de estudio o programa); es una entidad que está en cambio constante y que se nutre de experiencias, conocimientos y de los distintos actores que son parte de él (stakeholders). No siempre estos se desarrollan con un soporte e infraestructura adecuados, que permite las transformaciones en el aula, y con el apoyo necesario a los académicos, de modo que se generan trabas a los avances en el proceso de implementación curricular.

Para una adecuada gestión económica de las universidades es necesario conocer sus costos. Por tanto, se debe generar información financiera que permita tomar decisiones racionales a los administradores universitarios. El objetivo es que las universidades cuenten con recursos suficientes para el desempeño de sus funciones, en un contexto de autonomía creciente de dichas instituciones, rendición de cuentas, un control directo más débil por parte del Estado, financiación basada en indicadores del rendimiento, mayor diversificación del presupuesto, mayor competencia entre las instituciones y una fuerte orientación al mercado (Girotto et al., 2013).

Resulta evidente que la rendición de cuentas pública (accountability) de la gestión de las universidades se ha instalado en muchos países europeos y también en otras partes del mundo (Frolich et al., 2010). Puede ser interpretado como un intento de parte de los gobiernos de utilizar en forma más sistemática incentivos financieros e indicadores de desempeño, con el objetivo de controlar el comportamiento organizativo y por ende, mejorar la eficiencia y calidad de las instituciones. Sin embargo, aunque las nuevas exigencias y adaptaciones en las formas de gobernanza suponen mejorar el nivel de eficiencia y la flexibilidad, existen contradicciones, ya que coexisten mayores rigideces a la gestión universitaria (Girotto et al., 2013; Kim, 2008).

Desde la perspectiva de estudio de costos en las universidades se han desarrollado varios estudios vinculados con el diseño de sistemas de información contables (Araújo y Gonçalves, 2014) para dichas instituciones. Principalmente, desde el inicio de la década de los noventa, se consideran innovaciones en experiencias relacionadas con los principios del costeo basado en la actividad, conocido como ABC (Activity Based Costing), identificando las ventajas y desventajas de dicho sistema (Garcia y Del Rio, 2006). Un ejemplo destacable, citado por García (2008), es la publicación en España del documento denominado "Libro Blanco de los Costes en las Universidades", de 2007 que plantea la necesidad de contar con un sistema de costeo universitarios, usando herramientas de contabilidad analítica, control presupuestario y 
control de gestión en las instituciones que han de ayudar a la toma de decisiones en términos de detectar eficiencias e ineficiencias del sistema y así, poder reasignar recursos y mejorar los niveles de operación.

Así como resulta necesario analizar el costo de implantación de una determinada titulación en una universidad (García, 2008) y de las otras actividades de la institución, también es relevante hacer un estudio de mayor profundidad del modelo educativo que establece el marco curricular futuro. Bien se sabe que el coste más importante de las universidades es el gasto en recursos humanos, y que tiene un carácter fijo y constante en el tiempo dentro de una escala de operación, generando diferencias en la estructura de costo, plan de desarrollo y necesidades de financiamiento entre las instituciones, especialmente en carreras técnicas o con marcada presencia de laboratorios, coexistiendo niveles muy variados de complejidad académica y calidad (Améstica et al., 2014). Asimismo, no es menos importante poder detectar las posibles ineficiencias existentes, sobre todo si se está implementado un modelo educativo que supone demandas en distintos aspectos, especialmente los académicos, como por ejemplo: la mayor carga académica que refleje los tiempos reales destinados a los procesos de innovación curricular y evaluación de aprendizajes, espacios académicos que permitan compartir buenas prácticas docentes y estrategias de evaluación y por consiguiente, los programas de capacitación necesarios en estrategias de evaluación de aprendizajes y desarrollo de capacidades técnico-pedagógicas en los docentes para el trabajo en el aula y que suponen mayor cantidad de horas. Por lo tanto, significa un cambio de escala en el consumo del costo más importante de la institución: la disponibilidad de recursos humanos.

La instalación de un modelo educativo hace necesario la existencia de un plan progresivo que es costoso y complejo. Supone diseñar e implementar una trayectoria coherente (asignaturas, talleres, módulos, actividades prácticas, laboratorios) que debe seguir el estudiante para adquirir conocimientos, desarrollar habilidades y actitudes que le permitan lograr las competencias establecidas en el perfil de egreso. Con ello existirán necesidades de apoyo a este objetivo, como son los recursos humanos y materiales (en equipamiento, tecnología e infraestructura) acorde con los enfoques metodológicos que se adopten y consistentes con los diseños curriculares.

\section{METODOLOGÍA}

El trabajo se basó en información cualitativa y cuantitativa de una universidad pública y regional chilena, con 32 carreras de pregrado de distintas áreas según Tabla 1, que se encuentran en diferentes etapas de su proceso de renovación curricular al año 2014, siendo el proyecto estratégico para la institución coherente con su plan estratégico de desarrollo y su planes de mejoramiento por autoevaluación y acreditación. Para realizar la valorización económica de la renovación curricular se han utilizado secuencialmente seis instrumentos que se han adaptado a la realidad universitaria, obteniendo información relevante de cada carrera: i) análisis de la línea de corte para identificar situación sin y con proyecto, ii) construcción del flujo de caja relevante con la cuantificación de los beneficios económicos y costos de la renovación, iii) cálculo del valor actual de costos (VAC), iv) cálculo del costo anual equivalente (CAE), v) análisis de escenarios a través de simulación Monte Carlo, y vi) medir impactos en el punto de equilibrio económico de cada carrera.

\section{i) Análisis de línea de corte}

Con el fin de sistematizar los cambios por la renovación curricular se realiza un análisis destacando aquellos factores más relevantes que deben abordarse en términos incrementales, separando la situación sin y con proyecto. Dicha información se realiza en base a la revisión de documentación institucional y consultas a expertos y distintos actores de la comunidad que están involucrados en el nuevo diseño e instalación del proyecto, identificando actividades, inversiones y gastos recurrentes en el tiempo que sean cuantificables económicamente y que se ven modificadas con la instalación de la renovación curricular. Asimismo, se pueden encontrar factores que se ven minimizados y que podrían generar un ahorro.

\section{ii) Construcción del flujo de caja relevante}

En concordancia con el punto anterior, se construye un flujo de caja relevante adecuado a la realidad universitaria, con el fin de evaluar económicamente el impacto de la renovación curricular, siendo importante precisar, que no necesariamente el flujo para carrera es replicable a otra, ya que cada una de ellas tiene sus propias especificidades y su estructura de costos debe responder a sus propias necesidades. Otro elemento fundamental es reconocer aquellas inversiones que son transversales a varias carreras y costos operacionales compartidos en toda la universidad. Es importante precisar que la estructura para el flujo de caja es la misma, cualquiera que sea la fuente de financiación u objeto de estudio, con las especificidades propias del área en análisis. No obstante, cuando la organización objeto del estudio está en funcionamiento, o también llamada "en marcha", se deben incorporar criterios particulares que ayuden a determinar el impacto entre la situación sin proyecto (actual) y con proyecto (modificada) (Sapag y Sapag, 2007). 
Tabla 1. Carreras de pregrado

\begin{tabular}{|c|c|c|c|c|c|c|c|}
\hline Carrera & Área UNESCO & $\begin{array}{l}\text { Año } \\
\text { inicio }\end{array}$ & $\begin{array}{l}\text { Duración } \\
\text { (años) }\end{array}$ & $\begin{array}{c}\text { Años } \\
\text { acreditación }\end{array}$ & $\begin{array}{c}\text { Arancel } \\
(\text { base }=100)\end{array}$ & $\begin{array}{c}\text { Horas } \\
\text { docentes }\end{array}$ & $\begin{array}{c}N^{\circ} \\
\text { Matrícula }\end{array}$ \\
\hline Carrera 1 & Arte y Arquitectura & 1969 & 6 & 5 & 122,76 & 1080 & 577 \\
\hline Carrera 2 & Arte y Arquitectura & 1994 & 5 & 6 & 113,10 & 364 & 235 \\
\hline Carrera 3 & Tecnología & 1982 & 5,5 & 6 & 109,45 & 727 & 532 \\
\hline Carrera 4 & Ciencias Sociales & 1995 & 5 & 6 & 90,19 & 1714 & 474 \\
\hline Carrera 5 & Ciencias Sociales & 2001 & 5 & 5 & 109,45 & 775 & 96 \\
\hline Carrera 6 & Tecnología & 1982 & 6 & 4 & 113,10 & 1273 & 746 \\
\hline Carrera 7 & Tecnología & 1994 & 6 & 4 & 113,10 & 1474 & 577 \\
\hline Carrera 8 & Agropecuaria y Cs. Mar & 1982 & 5,5 & 4 & 113,10 & 505 & 83 \\
\hline Carrera 9 & Tecnología & 2004 & 6 & 0 & 113,10 & 782 & 394 \\
\hline Carrera 10 & Tecnología & 1990 & 5 & 5 & 113,10 & 1793 & 708 \\
\hline Carrera 11 & Tecnología & 2004 & 6 & 0 & 113,10 & 606 & 348 \\
\hline Carrera 12 & Tecnología & 1969 & 4 & 0 & 95,42 & 617 & 301 \\
\hline Carrera 13 & Tecnología & 1969 & 4 & 0 & 95,42 & 557 & 403 \\
\hline Carrera 14 & Tecnología & 1990 & 4 & 0 & 95,42 & 557 & 199 \\
\hline Carrera 15 & Tecnología & 1990 & 4 & 4 & 95,42 & 694 & 275 \\
\hline Carrera 16 & Ciencias Sociales & 1982 & 5 & 5 & 90,59 & 1902 & 506 \\
\hline Carrera 17 & Ciencias Sociales & 1991 & 5 & 4 & 113,10 & 2045 & 871 \\
\hline Carrera 18 & Tecnología & 1990 & 5 & 5 & 96,10 & 407 & 166 \\
\hline Carrera 19 & Salud & 1971 & 5 & 5 & 113,15 & 430 & 373 \\
\hline Carrera 20 & Salud & 1967 & 5 & 5 & 96,10 & 657 & 327 \\
\hline Carrera 21 & Salud & 2006 & 5 & 6 & 111,51 & 353 & 320 \\
\hline Carrera 22 & Arte y Arquitectura & 1969 & 5 & 5 & 105,99 & 460 & 166 \\
\hline Carrera 23 & Ciencias Sociales & 2006 & 5 & 5 & 118,38 & 1215 & 258 \\
\hline Carrera 24 & Educación & 2006 & 4,5 & 5 & 83,32 & 811 & 299 \\
\hline Carrera 25 & Educación & 1972 & 5 & 6 & 83,32 & 731 & 188 \\
\hline Carrera 26 & Educación & 2004 & 5 & 5 & 83,32 & 1309 & 173 \\
\hline Carrera 27 & Educación & 1966 & 5 & 6 & 83,32 & 779 & 266 \\
\hline Carrera 28 & Educación & 1972 & 5 & 5 & 83,32 & 737 & 196 \\
\hline Carrera 29 & Educación & 1970 & 4 & 6 & 83,32 & 935 & 130 \\
\hline Carrera 30 & Educación & 1967 & 4 & 5 & 83,32 & 665 & 178 \\
\hline Carrera 31 & Educación & 2010 & 4 & 3 & 83,32 & 1090 & 96 \\
\hline Carrera 32 & Educación & 2003 & 5 & 5 & 83,32 & 754 & 175 \\
\hline
\end{tabular}

La construcción del flujo de caja se proyecta desde el periodo actual (cero) hasta el número de periodos que entra en régimen o se estabiliza el proyecto, pudiéndose estimar metodológicamente por los años de la carrera renovada. Otra consideración importante, aunque en el corto plazo son de menor impacto, muchas carreras pueden generar algunos beneficios económicos (ingresos) en el corto plazo.

Ingresos

Ingreso por mayor oferta de vacantes. Se puede inferir que un nuevo programa renovado puede repercutir hacia los potenciales estudiantes, en el entendido que hace más atractiva una titulación, posibilitando un aumento de matrícula respecto a la actual situación. Por ello, se hace imprescindible reconocer económicamente los mayores ingresos que se podrían dar por este concepto.

Ingreso por mejora de la deserción. Entre los objetivos de la renovación curricular también se encuentra bajar las tasas de deserción. Ante un nuevo escenario, habría un número de estudiantes que podrían incrementar la matrícula, dada una mayor retención. 


\section{Ahorros}

Ahorro de costos por reconversión del recurso humano. Además de las nuevas asignaturas a dictar, existen algunas que se desechan. En consecuencia, ante un escenario de no reconversión del recurso humano académico, se podrían generar desvinculaciones de personal, por lo cual este hecho debe reconocerse económicamente.

Ahorro de costos por asignaturas. Durante el proceso de cambio curricular se generan dos hechos esenciales. Por un lado, el nuevo plan de estudio que significa mayores costos en la oferta de asignaturas. Y por otro lado, la continuidad del plan antiguo para aquellos que deben finalizar el programa actual. Este último hecho genera un ahorro a medida que van egresando los estudiantes.

\section{Costos}

Contratación a jornada completa de académicos. Dentro de los costos más importantes se encuentra la relacionada con las nuevas áreas a incorporar en el nuevo plan. Ejemplo de ello, asignaturas de ciencias básicas (matemática, biología, física y química), asignaturas de la profesión (especialidad y ámbitos específicos). Para tales efectos se debe estimar un salario acorde con el perfil requerido. Asimismo, el número de académicos a contratar debe hacerse paulatinamente en concordancia con los avances del nuevo plan de estudio.

Contratación de profesionales y técnicos de laboratorio. La incorporación de nuevas metodologías implican en algunos casos, la incorporación de laboratorios, talleres y/o labores que no existen. Con el nuevo plan de estudio se incorporan profesionales de apoyo académico, como técnicos laborales. Su cuantificación debe hacerse en concordancia con el avance curricular y con la planificación de las nuevas instalaciones, para el desarrollo de las áreas propuestas.

Contratación de funcionarios administrativos. En forma muy excepcional, producto de la incorporación de nuevas instalaciones, se necesita administrativos que tengan a cargo la atención de los estudiantes y el cuidado de la nueva infraestructura.

Costos operativos. Es importante reconocer los costos recurrentes que genera la instalación del nuevo modelo curricular, y que afectará los presupuestos de las escuelas y/o departamentos y que puede diferir entre una titulación y otra.

\section{Inversión}

Inversión en infraestructura. La renovación curricular hace necesario contar con infraestructura acorde al nuevo modelo (nueva y/o remodelada). En este ámbito, se debe cuantificar las inversiones que son transversales a todas las carreras que entran en este régimen.

Inversión en infraestructura de especialidad. Se debe costear la infraestructura de especialidad, acorde al avance curricular.

Inversión en equipamiento. Debe ponerse valor a la compra del equipamiento y equipos, tanto general como de especialidad, acorde al avance del plan de estudio.

Inversión en mobiliarios. Debe cuantificarse la compra de mobiliario y habilitación de la nueva infraestructura (nueva y/o remodelada), tanto general como de especialidad.

Inversión en licencias de software. Debe cuantificarse la compra de licencias de software (nuevas y/o renovación) para los estudiantes.

Inversión en bibliografía. Debe cuantificarse la compra de material bibliográfico y la adquisición de bases de datos.

Inversión en capacitación. Debe cuantificarse la inversión en capacitación a los académicos.

\section{iii) Cálculo del valor actual de costos (VAC)}

Dado que la renovación curricular, en general, se puede definir desde una perspectiva para la gestión universitaria como un "problema de costos" en el corto plazo y necesaria de financiar para su implementación y con el fin de evaluar económicamente el impacto a valor presente, se calcula el valor actual de costos (VAC), que es una extensión del criterio de evaluación del Valor Actual Neto (VAN), muy utilizado en finanzas (Sapag y Sapag, 2007; Zúñiga-Jara, Soria, y Sjoberg, 2011). Siendo una información fundamental para la toma de decisiones. 
Sin embargo, es preciso señalar que la evaluación de proyectos pretende medir, objetivamente una serie de magnitudes cuantificables que, mediante el uso de técnicas matemáticas, generan una serie de indicadores e información para la toma de decisiones. No debe ser considerada como una herramienta que, por sí sola, decida la ejecución del proyecto en función de su conveniencia económica, ya que existen otros factores de decisión, como son los de carácter político, social, moral y estratégico, entre otros, que pueden llevar a la realización o no del proyecto. En este sentido, el que sea un proyecto del área educativa puede llevar a un cuestionamiento de las técnicas a utilizar, dado que pertenecen al ámbito de la evaluación privada. Pero tanto la evaluación social y privada en términos generales, utilizan criterios más o menos similares, diferenciándose en la valoración que le dan a los costos y a los beneficios asociados al proyecto, convirtiéndose en un instrumento de uso prioritario para los agentes económicos encargados de la asignación de recursos de inversión, no importando la fuente de financiación, sea ésta pública o privada.

El valor actual de costos (VAC) se utiliza cuando los beneficios son difíciles de valorar, pero se reconoce que son deseables. Asimismo, cuando los beneficios de dos alternativas son idénticos y en ambos casos se busca alternativa de mínimo costo. Se expone su formulación en la ecuación (1):

$V A C=I_{0}+\sum_{t=1}^{n} \frac{C_{t}}{(1+r)^{t}}$

Siendo $I_{0}$ la inversión inicial en periodo cero; $C_{t}$ el costo en cada periodo $\mathrm{t} ; n$ el número de periodos (horizonte del proyecto, se excluye el periodo cero); y $r$ la tasa de descuento.

Para efectos metodológicos, dado que estamos trabajando sobre los datos de una universidad pública, usaremos tasa social de descuento r, coherente con lo propuesto por Sapag y Sapag (2007). En caso de una universidad privada, se sugiere utilizar el modelo CAPM (Capital Asset Pricing Model), ya que este criterio es utilizado preferentemente en la evaluación privada de proyectos (Zúñiga-Jara et al., 2011). El proceso de estimación del precio social del capital o tasa social de descuento, depende del enfoque metodológico que se adopte y los supuestos con relación a las variables explicativas que sugieren cada uno de ellos. En el caso chileno se obtiene el dato actualizado del Sistema Nacional de Inversiones en Chile, en base a la metodología de Harberguer que tiene un valor promedio de $7 \%$, con un rango que fluctúa entre un mínimo de 6,4\% y un máximo de 7,4\% (Ministerio de Desarrollo Social, 2013).

\section{iv) Cálculo del costo anual equivalente (CAE)}

Para determinar el impacto anual de las diferentes carreras, ya que éstas tienen distinto horizonte de evaluación asociado a su duración o periodo de implantación de la renovación curricular y con lo cual posibilita compararla entre sí, un indicador complementario a utilizar es el costo anual equivalente (CAE), para lo cual es necesario calcular inicialmente el valor actual de costos (VAC). A partir de la ecuación anterior, se puede obtener el costo anual equivalente (CAE) (Riggs et al., 2002), como una medida de comparación de costos, tal y como muestra la ecuación (2):

$$
C A E=V A C \frac{r(1+r)^{n}}{(1+r)^{n}-1}
$$

\section{v) Simulación Monte Carlo}

Para darle un sustento estadístico a los resultados obtenidos y obtener escenarios posibles. Los resultados del VAC y CAE se someten a la técnica de evaluación de riesgo mediante la simulación de Monte Carlo, utilizando el paquete estadístico @RISK 6.0 (Palisade) en el entorno Excel. Definidas las variables de entrada (salarios docentes, metros cuadrados a construir, valor económico de construcción, equipamiento, software, bibliografía, gastos operacionales y otros) y después de crear un modelo de simulación para los costos, se determinan las variables de salida para el análisis del VAC y CAE y se ejecutan iteraciones. Las proporciones se expresan con una probabilidad del $90 \%$.

\section{vi) Punto de equilibrio de cada carrera de pregrado}

Como un análisis complementario y dentro de las innovaciones metodológicas realizadas al aplicar herramientas económico-financieras a la realidad universitaria se propone calcular el "punto de equilibrio mínimo" de cada carrera, es decir, cuantificar la magnitud del cambio de costo de la situación nueva con renovación curricular con respecto a una situación base (sin renovación curricular), a partir de ciertas características comunes (Horngren et al., 2007). Sin embargo, esta herramienta es factible en la medida que las universidades cuenten con un modelo de costeo, identificando los costos directos e indirectos y por 
consiguiente el costo total por carrera o programa (Araújo y Gonçalves, 2014). Sin embargo, se puede hacer una aproximación a partir de los ingresos monetarios por cada carrera (también llamadas arancel, tasas 0 precio), con el fin de obtener el número de estudiantes que debería necesitar cada carrera para solventar el aumento de costos por la renovación.

El análisis de punto de equilibrio, aunque no es una técnica para evaluar la rentabilidad económica del proyecto, si es efectivo para realizar un análisis de sensibilidad económica, ya que permite realizar modificaciones en los ingresos por unidad y en la estructura de costos (aumentarlos y disminuirlos) para ver su impacto en el punto de equilibrio en la universidad como un todo, para lo cual es posible ponderar por el número de matrícula de cada carrera.

\section{RESULTADOS Y DISCUSIÓN}

A partir del reconocimiento de los costos que involucra cada renovación curricular, para cada una de las 32 carreras de pregrado, se construyó el flujo de caja respectivo, tomando en cuenta el periodo de egreso de cada titulación como horizonte del proyecto. En virtud de este cálculo, se pudo sistematizar la Tabla 2, de máximos y mínimos del VAC, CAE, impacto en términos de punto de equilibrio para cada una de las 32 carreras de pregrado con una probabilidad del $90 \%$ según simulación Monte Carlo. De los factores que inciden en el costo de la renovación curricular, podemos señalar que en promedio para todas las carreras el costo docente alcanza un $47 \%$, seguido por la inversión con fines generales con un $25 \%$, inversión especializada con un $20 \%$ y costos de operación con un $9 \%$. La carrera con mayor impacto de costo docente es del área de Tecnología de duración 6 años con un 57\% y la con menor incidencia es del área de Arquitectura y Artes de duración 5 años con un 38\%. Al sumar la inversión general y especializada el impacto promedio es de un $44 \%$ en costo total.

De los cálculos obtenidos en la simulación Monte Carlo, se puede observar que el costo total a valor actual para la institución en base a las 32 carreras alcanza a los M\$10.013.986,2. Sin embargo, esta aproximación se hace en base a la proyección de inversiones y gastos en un momento del tiempo, sabiendo que las carreras gradualmente han entrado e ingresarán a este proceso en distintos momentos del tiempo. A nivel de individual, la carrera con el Valor Actual de Costos (VAC) promedio alcanza a M\$312.937,1 donde valor máximo alcanza a M\$570.789,0 de una carrera del área de Tecnología de 6 años de duración y con un valor mínimo M\$164.001,6 una carrera del área de Educación de 4 años. La desviación estándar del VAC entre las carreras fluctúa entre $\mathrm{M} \$ 34.553,4$ y M\$5.427,7. Al revisar la inversión per cápita por estudiante se puede observar que se mueve en un rango de un mínimo de $M \$ 380,3$ de una carrera del área de Ciencias Sociales de 5 años y un máximo M\$3.339,1 en carrera del área de Agropecuaria y Cs. del Mar de 5,5 años de duración, con una desviación estándar de $M \$ 671,6$ y que el promedio universidad por estudiante es de $\mathrm{M} \$ 1.194,6$.

En tanto, el Costo Anual Equivalente (CAE) como medida para comparar el impacto anual promedio alcanza a $\mathrm{M} \$ 75.387,4$ donde valor máximo alcanza a $\mathrm{M} \$ 119.749,1$ y con un valor mínimo $\mathrm{M} \$ 47.617,2$. Al revisar el coeficiente de correlación entre años de duración de la carrera y VAC es de 0,865 , con un $\mathrm{R}^{\wedge} 2$ ajustado de 0,74 , evidenciando una relación directa entre los recursos necesarios y la duración de la carrera en el periodo implementación de la renovación. En tanto, el coeficiente de correlación entre número de matrícula y VAC es de 0,568, con un $\mathrm{R}^{\wedge} 2$ ajustado de 0,30. Al revisar el coeficiente de correlación entre años de acreditación de la carrera y VAC es de 0,026, con un $\mathrm{R}^{\wedge} 2$ ajustado de $-0,03$. En concordancia con los resultados anteriores, al agrupar las carreras por área Unesco según Figura 1 y comparar su costo promedio, se puede establecer que las carreras del Área de Arquitectura y Artes presentan el mayor costo (VAC) con un M\$ 406.933,8 y un CAE de M\$ 93.066,3. En tanto, las carreras del Área Educación presentan un menor VAC de M\$211.458,4 y un CAE de M\$55.185,4.

Bajo la premisa de que el financiamiento para solventar el costo de la renovación curricular se tenga que realizar a partir de los precios que se cobran por cada una de la carreras (arancel), se estima un punto de equilibrio expresado por el número de estudiantes matriculados en términos de su aporte económico. En base a los resultados obtenidos podemos señalar que en promedio se necesitan incrementar a lo menos 31,7 estudiantes (CAE (M\$) /Arancel carrera) para solventar los costos y al dividir por el número de años de cada carrera (Punto equilibrio incremental), se obtiene un promedio de 6 estudiantes por cohorte. La carrera con mayor impacto alcanza a 9 estudiantes por cohorte del Área de Educación de 4,5 años y la con menor exigencia es de 4 estudiantes por cohorte del Área de Agropecuaria y Ciencias del Mar de 5,5 años de duración. 


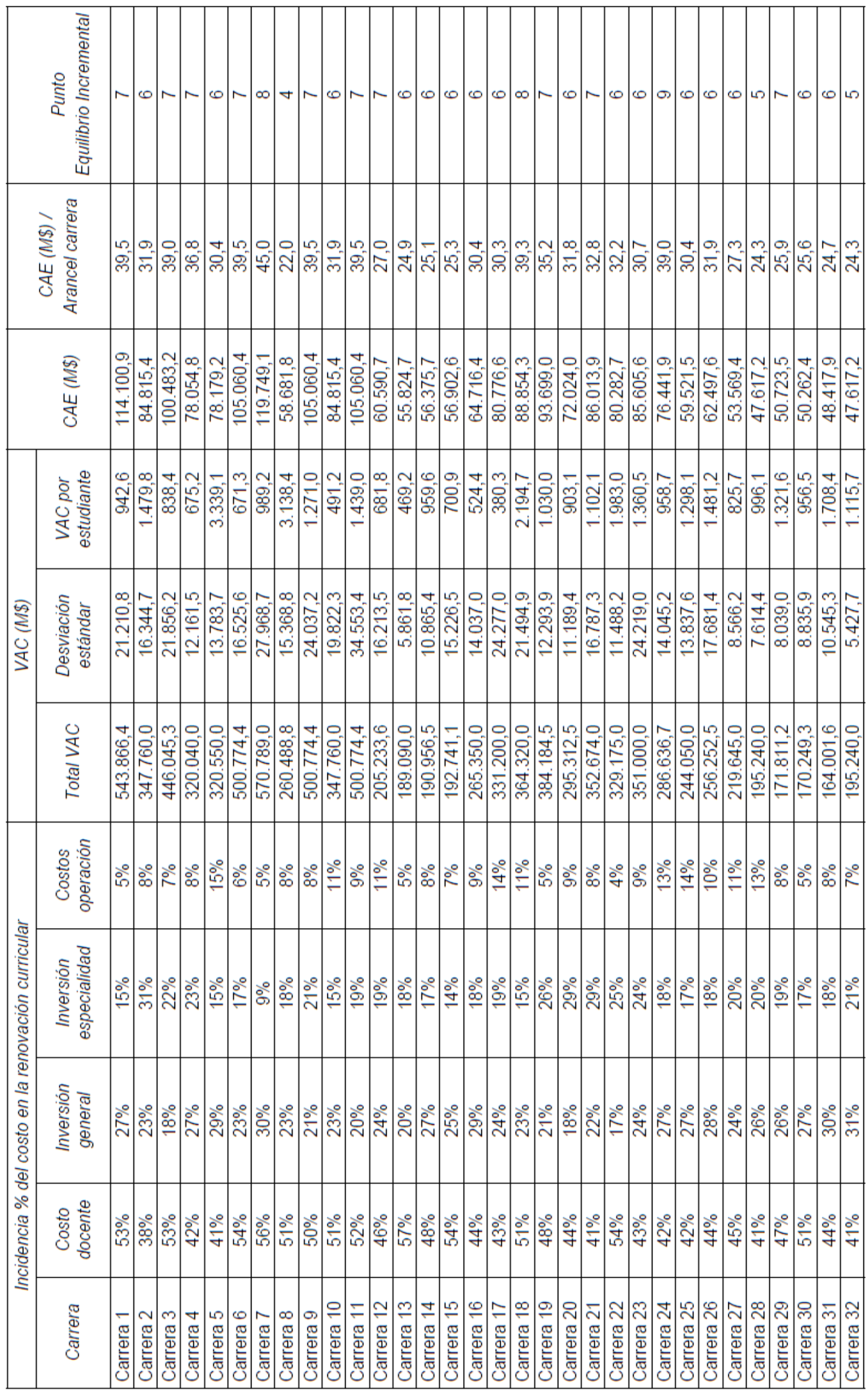




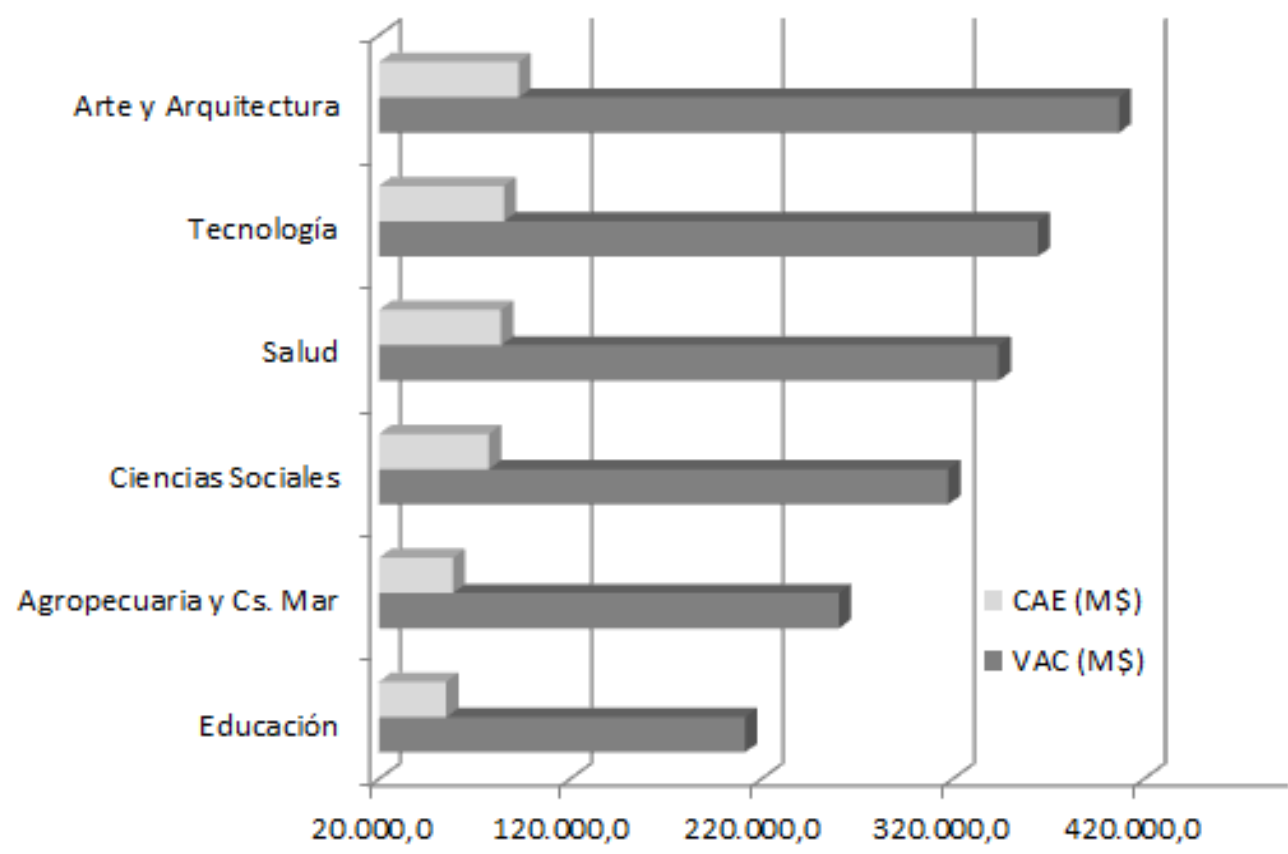

Fig. 1: Costo por área de conocimiento Unesco. Expresado en CAE (Costo Anual Equivalente) y VAC (Valor Actual de Costos).

Al cuantificar las inversiones y costos se pudo establecer que cada carrera se encuentra en estados de desarrollo distinto, siendo la renovación curricular la mejor justificación u oportunidad para algunas carreras de poder revertir sus deficiencias iniciales e históricas. Otro elemento visible, es la idea de la reconversión de la planta académica, pero al analizar los impactos económicos se pudo establecer que es un factor difícil de llevar acabo, generando al final un incremento de mano de obra docente. Tal como señaló y producto de la renovación curricular, la universidad necesitará a lo menos seis estudiantes matriculados por cohorte, ya sea vía nuevos ingresantes o mejora de la retención, aunque ésta última no es muy clara. Lo anterior se alivia en la medida que el Estado realice un aporte extraordinario de recursos para estos fines, situación que se evidencia en muchas universidades a través de fondos competitivos. Sin embargo, no hay que perder de vista, cualquiera sea la forma de financiación y más aún en un escenario que propicia la "gratuidad", Chile debe avanzar hacia un sistema en que los aranceles reflejen el real costo (y calidad) asociado a las actividades que recibe directamente el estudiante (Espinoza y Urzúa, 2015).

\section{CONCLUSIONES}

De los resultados mostrados, de su análisis y de su discusión se pueden obtener las siguientes conclusiones: (1) la renovación curricular es un hecho complejo para cualquier universidad, y el avanzar más rápidamente depende no sólo de disponer de un buen proyecto alineado con su misión institucional, sino de disponer de los recursos necesarios para su implementación en la cantidad y plazos necesarios, para asegurar la sustentabilidad académica y financiera de la institución. (2) La evaluación económica de una carrera de pregrado puede diferir una de otra, dada sus propias especificidades, por lo tanto, es imposible inferir que el costo obtenido para la renovación curricular de una carrera es transferible a otra en su totalidad, siendo necesario evaluarse independientemente. (3) Una financiación eficiente es uno de los objetivos centrales que se pretende alcanzar en el sistema universitario, haciéndose imperativa la financiación de los modelos educativos que aseguren el éxito y, a su vez minimicen las necesidades económicas, en un contexto de racionalización de la gestión económica de las universidades.

\section{REFERENCIAS}

Améstica, L., Gaete, H., y Llinàs-Audet, X., Segmentación y clasificación de las universidades en Chile: desventajas de inicio y efectos de las políticas públicas de financiamiento, DOI:10.4067/S071833052014000300009, Ingeniare. Revista Chilena de Ingeniería, 22(3), 384-397 (2014)

Araújo, M., y Gonçalves, R., Costing Systems for Use in Public Universities: the Brazilian and international context, http://www.ijern.com; ISSN: 2201-6740, International Journal of Education and Research, 2(12), 1$12(2014)$ 
Clark, B. R., The entrepreneurial university demand and response, DOI:10.1080/13583883.1998.9966941, Tertiary Education and Management, 4(1), 5-15 (1998)

Clemente-Ricolfe, J., y Escribá-Pérez, C., Análisis de la percepción de las competencias genéricas adquiridas en la universidad, DOI:10.4438/1988-592X-RE-2013-362-241, Revista de Educación, 362, 535$561(2013)$

Díaz, A., El enfoque de competencias en la educación. ¿Una alternativa o un disfraz de cambio?, http://www.iisue.unam.mx/; ISSN 2448-6167, Perfiles Educativos, XXVIII(111), 7-36 (2006)

Espinoza, R. y Urzúa, S., Las Consecuencias Económicas de un Sistema de Educación Superior Gratuito en Chile, DOI: 10.4438/1988-592X-RE-2015-370-295, Revista de Educación, 370,10-44 (2015)

Frolich, N., Kalpazidou, E., y Rosa, M., Funding systems for higher education and their impact on institutional strategies and academia: A comparative perspective, DOI:10.1108/09513541011013015, International Journal of Educational Management, 24(1), 7-21 (2010)

García, M., Algunos aspectos sobre la racionalidad en la financiación universitaria. DOI:10.4438/1988-592X0034-8082-RE, Revista de Educación, Número ext, 267-284 (2008)

Garcia, T., y Del Rio, R., Development and Implementation of a University Costing Model, DOI: 10.1111/j.1467-9302.2006.00533.x, Public Money and Management, 26(4), 251-255 (2006)

Girotto, M., Mundet, J., y Llinàs-Audet, X., Estrategia en la universidad : ¿cuestión de calidad, gerencialismo y relaciones político-financieras?, DOI:10.4438/1988-592X-RE-2011-361-136, Revista de Educación, 361, 95-116 (2013)

González González, J. M., Arquero Montaño, J. L., y Hassall, T., Consolidación de la formación por competencias en la Universidad española: estudio de un caso, DOl:10.5944/educxx1.17.2.11483, Educación XX1, 17(2), 145-168 (2014)

González, J., y Wagenaar, R., Tuning Educational Structures in Europe. Informe Final, Universidad de Deusto y Universidad de Groningen, 339 p., Bilbao, (2003)

González, V., y González, R., Competencias genéricas y formación profesional: un análisis desde la docencia universitaria, http://rieoei.org/; ISSN-e 1022-6508 Revista Iberoamericana de Educación, 47, 185-209 (2008)

Guzmán, M., Maureira, O., Sánchez, A., y Vergara, A., Innovación curricular en la educación superior ¿Cómo se gestionan las políticas de innovación en los (re)diseños de la carreras de pregrado en Chile? http://www.iisue.unam.mx/perfiles; ISSN 2448-6167, Perfiles Educativos, XXXVII(149), 60-73 (2015)

Hoogveld, A., Paas, F., y Jochems, W. Application of an instructional systems design approach by teachers in higher education: individual versus team design, DOI: 10.1016/S0742-051X(03)00055-6, Teaching and Teacher Education, 19(6), 581-590 (2003)

Horngren, C. T., Datar, S. M., y Foster, G., Contabilidad de costos: un enfoque gerencial, 12th ed., Pearson Ed., México. (2007)

Icarte, G., y Lávate, H., Metodología para la Revisión y Actualización de un Diseño Curricular de una Carrera Universitaria Incorporando Conceptos de Aprendizaje Basado en Competencias, DOI:10.4067/S0718-50062016000200002, Formación Universitaria, 9(2), 3-16 (2016)

Ion, G., y Cano, E., La formación del profesorado universitario para la implementación de la evaluación por competencias, DOI:10.5944/educxx1.15.2.141, Educación XX1, 15(2), 249-270 (2012)

Kim, T., Changing University Governance and Management in the UK and Elsewhere under Market Conditions: Issues of Quality Assurance and Accountability, http://www3.mruni.eu/; ISSN 1822-8038, Intellectual Economics, 2(4), 35-42. (2008)

Kirkpatrick, J., y Hawk, L., Curricula and Evaluation: Maximizing Results. Training y Development, 60(6), 6162. (2006) 
López, E., En torno al concepto de competencia: un análisis de fuentes, http://recyt.fecyt.es/; ISSN 1989639X. Profesorado. Revista de Currículum y Formación del Profesorado, 20(1), 311-322 (2016)

Márquez, M., Sandoval, J., Torres, M., y Pavié, S., Estudio de caso de la coherencia interna de programas de asignaturas en seis carreras que promueven competencias de la Universidad Austral de Chile, http://mingaonline.uach.cl/; ISSN 0718-0705. Estudios Pedagógicos, XXXVI(2), 117-133 (2010)

Martensen, A., y Grønholdt, L., Quality in higher education: linking graduates' competencies and employers' needs, DOI:10.1108/17566690910, International Journal of Quality and Service Sciences, 1(1), 67-77. (2009)

McChlery, S., McKendrick, J., y Rolfe, T., Activity-Based Management Systems in Higher Education, DOI: 10.1111/j.1467-9302.2007.00602.x, Public Money y Management, 27(5), 315-322 (2007).

Ministerio de Desarrollo Social, Estudio de Actualización del Modelo de Estimación de la Tasa Social de Descuento en el Marco del Sistema Nacional de Inversiones de Chile. Santiago, Chile. (2013)

Quevedo-Blasco, R., Ariza, T., y Buela-Casal, G., Evaluación de la satisfacción del profesorado de ciencias con la adaptación al espacio europeo de educación superior, DOI:10.5944/educXX1.18.1.12311, Educación $X X 1,18(1), 45-70(2015)$

Reich, R., Machuca, F., López, D., Prieto, J.P., y Rodríguez-Ponce, E., Bases y desafíos de la aplicación de convenios de desempeño en la educación superior de Chile Basis and challenges of the application of performance agreements in Chilean higher education, DOI:10.4067/S0718-33052011000100002, Ingeniare, Revista Chilena de Ingeniería, 19(1), 8-18 (2011)

Riggs, J.L., Bedworth, D.D., y Randhawa, R.U., Ingeniería Económica, Cuarta edición, Alfaomega Ediciones, México. (2002)

Sapag, N., y Sapag, R., Preparación y evaluación de proyectos, Quinta edición, McGraw Hill, México. (2007)

Schmal, R., y Ruiz-Tagle, A., Una metodología para el diseño de un currículo orientado a las competencias, DOI:10.4067/S0718-33052008000100004, Ingeniare. Revista Chilena de Ingeniería, 16(1), 147-158 (2008)

Solé, F., y Llinàs-Audet, X., De la burocracia profesional a la tecnópolis : los desafíos estratégicos de la gestión universitaria. DOI:10.4438/1988-592X-0034-8082-RE, Revista de Educación, 355, 17-30 (2011)

Tejada, J., La alternancia de contextos para la adquisición de competencias profesionales en escenarios complementarios de Educación Superior: marco y estrategia. DOI:10.5944/educxx1.15.2, Educación XX1, 15(2), 17-40 (2012)

Zúñiga-Jara, S., Soria, K., y Sjoberg, O., Costo del capital y evaluación de proyectos en Latinoamérica: una clarificación, http://www.fce.unal.edu.co; ISSN 0121-5051, Innovar, 21(41), 39-50 (2011) 